sans être amusant, ce qui me donne du remords de ne pas le trouver tel. Il est fort estimé ici moralement et fort connu. Sa belle-mère qui est restée avec lui (grand avantage pour tous deux) en parle comme du plus excellent des hommes....

Ainsi de CARro était ennuyeux! Il se prenait sans doute trop au sérieux, s'appesantissait dans ses récits, se donnait de l'importance. Mais, ses emballements et ses fanfaronnades mis à part, c'était un homme dévoué et sincère; fat, snob, dirions-nous aujourd'hui; intéressé, il est vrai, mais honnête; adroit, pas bête, mais pas très intelligent non plus. Sa plus grande chance fut de rencontrer sur sa route, au début de sa carrière, la vaccination et de s'y consacrer avec un zèle et une adresse qui lui valent la reconnaissance très méritée de la postérité.

\title{
Francesco Camuzios Consilium über das Steinleiden
}

\author{
Von Charles Salzmann, Zürich
}

In der Manuskriptensammlung der Zentralbibliothek Zürich findet sich in Mscr. Sim. 204 $\mathbf{c}^{1}$, einem medizinischen Sammelband aus dem Besitze Conrad Gessners, ein Consilium des Luganeser Arztes Francesco Camuzıos, zusammen mit anderen ähnlichen Consilia gegen das Steinleiden, Abschriften von unbekannter Hand. Diese lateinische, undatierte Abschrift, als ein Consilium abgefaßt, dürfte einer früheren Zeit angehören, vor 1550. Der Sammler dieser Schriften ist unbekannt, noch viel weniger weiß man, wer sie C. Gessner schenkte. Die erwähnte Sammlung von Ratschlägen gegen das Steinleiden enthält folgende Beiträge: zwei vom Graf von Solms, eine von Casarate «uff Italia», eine von Hieronymus Graf von Rapperswil, jene des Francesco Camuzio, und eine von Vadian ${ }^{2}$. Aus dieser Zusammenstellung kann man annehmen, daß der Sammler im ostschweizerischen oder süddeutschen Raume zu suchen wäre. Im dritten Bande des Thesaurus Medicinae Practicae (Mscr. Sim. 204 c) sammelte C. Gessner neben eigenen Notizen, Krankengeschichten, Konsilien, Rezeptarien, Be-

${ }^{1}$ Mscr. Sim. 204 c Z. B.Zch.

${ }^{2}$ B. Mrte, Ein Konsilium Vadians, Schw. Med. Wschr. 62. Jg. (1932), Nr. 11. Herrn Prof. Dr. B. Mrut bin ich für den Hinweis auf den Camuzio-Text und manche Angaben zu Dank verpflichtet. 
handlungsanweisungen vom Kopf bis zu Fuß, angelegt im Hinblick auf eine spätere Veröffentlichung.

Belege, ob C. Gessner mit den Camuzio in Verbindung gestanden, lassen sich keine erbringen ${ }^{3}$. Mit Jон. Muralto und Th. Duno ${ }^{4}$ trat GeSSNER erst in Zürich nach 1555 in nähere freundschaftliche Beziehungen. Wohl aus früheren Zeiten, vielleicht anläßlich seiner im Sommer 1544 unternommenen Reise nach Venedig, kannte C. Gessner Ärzte aus den ennetbirgischen Landvogteien, wie dies aus seinem Brief an FABRIcius in Chur vom 22. September 1559 hervorgeht. Hier bittet er FABRICIUS, dem Briefüberbringer, einem «armen Jüngling», durch den Arzt Hieronymus BrIxIUS einen italienischen Empfehlungsbrief schreiben zu lassen an den «trefflichen» QUADRI und seinen Sohn, damit sie dem jungen Manne helfen, den schwarzen Helleborus und andere Pflanzen zu suchen: «denn ich höre, daß der Berg Cenere, nahe bey Lugano reich an den seltesten Gewächsen sey». Vermutlich handelt es sich um die Luganeser Chirurgen BatTista Quadrio und dessen Sohn Bonifatio, die beide noch 1597 in den «Atti della visita del vescovo Arichinti» als «Barbieri all'ospedale di Santa Maria di Lugano» aufgeführt werden.

Von Francesco Camuzio sind nur wenige Angaben erhalten. Der «Consiglio communale del Borgo» beschließt seinem «medico magister» 1512 eine Gratifikation: «per le molte pene usate nel disimpego dell'arte sua a favore di Lugano, siccome l'unico medico ivi assunto in esercizio communale» (E. MоттA ${ }^{5}$ ). Er entstammte einer alten Ärztefamilie aus Como und Lugano, Nachfahr eines Humanisten, der zu Anfang des 15. Jahrhunderts im Dienste Filippo Maria Viscontis gestanden ${ }^{6}$. Für seinen Vater Ludovico stiftete Francesco Camuzio die bekannte Camuzio-Kapelle in der Chiesa Santa Maria degli Angeli in Lugano, die er um 1520 mit den heute noch erhaltenen Fresken, vermutlich durch Bramantivo, ausschmücken ließ ${ }^{7}$. Daselbst bezeugt ein Gedenkstein: Hic situs est Ludovico / Camucius

${ }^{3}$ J. Hanhart, Conrad Gessner, Winterthur 1824, p. 244-6.

${ }^{4}$ C. Salzmann, Thaddeo Duno von Locarno als Stadtarzt in Zürich, Vjschr. Naturf. Ges. Zürich, Jg. 85 (1940).

${ }^{5}$ E. MotтA, Bibliografia medica della Svizzera italiana. Bellinzona 1887, p. 9/10.

- Pier Candido Decembrio, Leben des Filippo Maria Viscontiu, Taten des Francesco Sforza, übersetzt und eingeleitet von Pн. FunK, Jena 1913, p. 43. - Boll. stor. della Svizzera italiana 1881, p. 189. - Hist.-Biogr. Lex. Bd. 2 p. 483.

${ }^{7}$ W. SuIDA, Tessiner Maler des beginnenden Cinquecento und ihre Beziehungen zu Bramantino. - Beltrami, Boll. stor. della Svizzera italiana 1893, p. 5/6. - V. ChIesa, L'ospedale civico di Lugano, Bellinzona-Lugano 1944, p. 28. 
chirurgicus / quondam clarus / Francisco fratribus / Il filio superstite / qui parens titulum pos. / MDXX.

Im übrigen liegt das Leben Francesco Camuzios im völligen Dunkel; um so heller aber erstrahlt das seines Sohnes Andreas, des bedeutendsten Tessiner Arztes des 16. Jahrhunderts.

Die Söhne Francescos (Andrea und Girolamo) studierten an der Universität Pavia, wo sich ANDrea den Ruf eines vortrefflichen Arztes, Humanisten und Theologen erwarb. Mit Girolamo Cardano (1501-1576) ${ }^{8}$ verfeindete er sich in einer Streitschrift wegen der Autorität Galens, die Cardano in einer Apologia zu widerlegen versuchte ${ }^{9}$. Der streitbare Geist andrea Camuzios erhob sich auch gegen Crato von Krafftheim (1519 bis 1586) über das Ausmaß des Aderlasses ${ }^{10}$. Auf dem Religionsgespräch zu Locarno am 4. August 1549 traten die Brüder Camuzio gegen Beccaria und die Locarner in die Schranken ${ }^{11}$. Andrea dozierte Philosophie anfangs in Como, später in Pavia, wo er Thaddeo Dunos Lehrer war, in Pisa als Professor der Medizin und Physik. 1557 erwarb er das Bürgerrecht von Mailand, wo er zeitweilig wirkte. Nach Giovio ${ }^{12}$ beteiligte er sich ärztlich und theologisch am Konzil von Trient (1545-1563). Kaiser Maximilian II. ernannte ihn 1564 zum Leibarzt, in dessen Diensten er in Wien und Mailand bis zu seinem Tode 1578 verblieb.

Das Folioblatt enthält in einer feinen Schrift wohlgeordnet nachfolgenden Text:

${ }^{8}$ H. Hefele, Des Girolamo Cardano von Mailand (Bürger von Bologna) eigene Lebensbeschreibung, übertragen und eingeleitet von H. HeFele, Jena 1914, p. 183. Nach seinem Vita propria vertrat Cardano ein Lehramt in Pavia in den Jahren 1540-42, 1544/45, 1559-62.

9 A. Camutir, Disputationes, quibus Hieronymi Cardani magni nominis viri conclusionem infirmantur, Galenus ab ejusdem in injuris vindicatur Hyppocratis praeterea aliquot loca diligentius, multo quam nunquam alias explicantur. Pavia 1553, p. 4.0. - H. CardanI, Medici ad Andream Camutium medicum Apologia, in Ars curandi parva ..., Tomus I, Basileae 1564, p. 80.

${ }^{10}$ A. von Haller, Bibl. med. pract. Vol. II, p. 146. K. Sprengel Pragmatische Geschichte der Arzneikunde, Halle 1801, 3. Teil, p. 257.

11 F. MeYer, Die evangelische Gemeinde in Locarno, Zürich 1836, 1. Bd. p. 190-3, 254.

12 Grovio, cit. n. c. TrezzinI: His. biogr. Lex. Bd. 2, p.483/4. 
Calculus renis. (Von einer andern Hand geschrieben.)

\section{Zum Stein der Nieren}

Camutis. Dis hat mir der verrümpte Doctor Franciscus Camutius Medicus, Luganensis der zu Meiland und anderswa hochgeacht, und von vil Herren beschickt wirt.

Ut praeserveris a Renum Calculo, haec servanda sunt circa victus regimen. Ut cibaria sint boni chymi, concoctu facilis, moderate sumpta horis debitis et consuetis.

Huius facultatis sunt.

Carnes Gallinarum, Caponum, Hedorum, Vitulorum lactentium, Castratorum iuvenum, Perdicum, Fasianorum, et avium parvarum in arboribus degentium.

Dammantur vero.

Aves aquaticae, Lac, Caseus, et Lacticinia omnia. Pisces non squamosi, lacunales et ex malis aquis vitandi, ut Anguillae, Tincae, et consimiles. Et fercula de farina, et pasta, Triticum coctum omnisque generis Leguminum et frumentaecorum usus. Et partes pingues animalium, extremae et interiores, ut intestina, Cerebra Hepata (pullorum exceptis), Splenes, Pulmones, Linguae, Corda, et Testes (exceptis Galli cantare et coire incipientis).

Crapula et mala concoctio ciborum fugienda. Abstinendum quoque a cibis crassis.

Aequandum quod comedit, in substantia, quantitate, qualitate, et ordine, et alijs, ut digestio bona fiat. Id lapidis generationem prohibet, dicit AvIGENNA.

Coitus fiat raro, et non a cibo immediate. Studeatque quotidie habere Ventris beneficium.

Nimis calida cibaria et acria, ut Cepae Allium, Porra, aestatis tempore potissime relinquantur.

Exercitium sit mediocre. Laboriosum, et a cibo, malum, illud maxime in quo Renes laborant, ut in Equitatione incommoda fit.

Cavendum quoque a strictura cinguli, et pannorum copia super Renes, et aliorum quae possint Renes calfacere.

Vina crassa, dulcia, pñtica (eine andere Hand schrieb darunter nochmals pontica ungekürzt), mala, Aer malus quoque, Ira, Furor, Tristitia evitentur.

Et hoc propono victus regimine. Servando adhibeatur diligentia, quia ex observatione eius magis praeservabitur quam per Medicinalia. Et si non 
servetur, erit corruptio ex eis maior, quam iuvamentum ex Medicinis convenientibus, Quia comesta et bibita quotidie sumuntur.

Quod si ex inobservantia dicti regiminis, vel propter aliam causam, materia in Corpore generatur, Tunc insurgit duplex intentio. Una ad causam materialem, quae est Pituita.

Altera ad agentem, quae est Caliditae Renum. Et quando nos (dicit Serapio) utramque causam generationis lapidis, materialem scilicet et agentem abscindimus et destruimus, tunc et lapidis generatio desinit.

Pro prima vero intentione, sententia Doctorum omnium et experientia est Vomitus a cibo, pluries in mense reiteratus, Evacuando Pituitam, cum aversione a Renibus. Et sepe in aestate in principio comestionis comedere de Cassia, et hyeme sumere de Resina Laricis electa, Ut natura assuefiat transmittere materiam per intestina, avertendo a Renibus. Et bis vel ter in mense aestate sumatur hoc.

R/ Floris Cassiae ऊ.s.

Pulveris Liquiritiae Novae rasae 3. s. Seminum Anisi

Malvae, Altheae, ana 7.1. Fiant boli, quos capiat hora caenae, cum panatella, paulo post.

Et mane sequenti bibat, iusculum brodij Carnium, decoctarum Cicerum Rubeorum Liquiritiae novae, Seminum Melonum et Radicis Graminis cum Zuccharo albo et nucleis Persicorum.

Nucleos et gummi cerasorum aliqui laudant (steht hier am Rande des Textes von zweiter Hand hingeschrieben).

Hyeme vero utatur et sumat de mane loco Cassia, Electuarium quantitate nucis juglandis Quod fiat ex Laricis Resina bene clara, cum pulvere ossium Mespilorum et semine Foeniculi non dulcis.

Caliditas vero Renum, fugiendo omnia supradicta calorum facientia remittitur. Ungantur quotidie loca Renum, oleo Violarum, Cucurbitae, Nemofarini, et Ungento albo GALENI pluries frigida Loto.

Haec sunt ad praeservationem valde utilia et experta.

Quando vero Calculus aderit, et doloribus infestabit, curationem exigit quam in praesentia non scribo. etc.

(Unter dem Text stehen noch einige Worte, von denen sich nur balnei, clysterum, cucurbitularum lesen lassen.) 
In freier Fassung besagt der Lateintext:

Bishar (soweit) D. CAmutius.

Um Nierensteine zu verhüten, schlage ich vor, ungefähr dieser Lebensweise zu folgen, weil nur fein gekochte Speisen, mäßig bei Zeiten eingenommen, gut verdaulich sind.

Dazu eignet sich das Fleisch von Hühnern, Kapaunen, Zicklein, Milchschweinen, Kälbern, Rebhühnern, Fasanen und kleinen Vögeln.

Dagegen schädlich sind: Wasservögel, Milch, Käse, alle milchhaltigen Speisen, schuppenlose Fische und Seefische aus verunreinigten Gewässern, wie Aale, Schleie und ähnliche; ferner Mehlspeisen, Milchsuppen, nebst jeder Art gekochtem Getreide und Gemüse, alle tierischen Fette, Eingeweide, Hirn, Leber (ausgenommen von Hühnern), Milz, Lungen, Herz und Hoden (ausgenommen von jungen Hähnen).

Übermäßiges Trinken und ungenügende Zubereitung der Gerichte muß vermieden werden, auch soll man sich von allzu fetten Speisen enthalten.

Beim Essen soll man Maß halten an Menge, wie in guter Auswahl und Speisefolge, um gut zu verdauen.

Dies verhindert die Steinbildung, sagt Avicenna.

Der Coitus erfolge selten und nicht nach dem Essen.

Täglich trachte man auf eine gute Verdauung.

Allzu warme und gewürzte Speisen mit Zwiebeln, Lauch müssen in der warmen Jahreszeit vermieden werden.

Man halte $\mathrm{Ma} \beta$ in körperlichen Bewegungen, ebenso ist jede Geschäftigkeit nach der Mahlzeit von Übel, wie Reiten, weil sie die Nieren dadurch anstrengen.

Auch soll man sich hüten vor zu straff angezogenem Gürtel und vor zu vielen Kleidungsstücken um die Hüfte und alles andere was die Nieren erhitzt.

Dicke, süße griechische Weine (pontica vina) soll man meiden, ebenso schlechte Luft, sowie Zorn, Wut und Traurigkeit.

Diese Lebensweise empfehle ich, weil sie bei verständnisvoller Anwendung mehr vermag als Medikamente; bei Nichtbefolgen vergrößert sich das Übel, um so mehr, als helfende Heilmittel vermögen, da Speisen und Getränke täglich eingenommen werden.

Daher vermehrt sich bei Nichtbefolgen des besagten Regimes oder aus anderer Ursache die Materia im Körper, dann entsteht eben eine doppelte Disposition, die eine zum festen Stoff, dies ist der Schleim, die andere zur Förderung, dies ist die Erhitzung der Nieren. 
Und wenn wir, sagt SERAPION ${ }^{13}$, beide Komponenten der Steinbildung, nämlich die stoffliche und die treibende, verhindern und vermeiden, dann bleibt auch die Steinbildung aus.

Was die erste Disposition angeht, soll man nach der Meinung und Erfahrung aller Ärzte pro Monat mehrmaliges Erbrechen veranlassen, den Schleim entleeren, um ihn von den Nieren abzuhalten.

Und oft soll man zur Sommerszeit vor der Mahlzeit Cassia ${ }^{14}$ einnehmen, im Winter aber klares Lärchenharz ${ }^{15}$, damit die Natur sich gewöhne, die Materia dem Darm zu übergeben unter Umgehung der Nieren.

Zwei bis dreimal soll man folgende Arznei in den Sommermonaten einnehmen:

Rp. Floris Cassiae $\tilde{3} . \mathrm{s}$.

Pulveris Liquiritiae Novae rasae 3. s. Seminum Ansi.

Malvae, Altheae ana 7.1. Fiant boli, quos capiat hora caenae, cum panatella paulo post.

Am folgenden Morgen trinke man einen Becher Fleischbrühe mit roten Kichererbsen ${ }^{16}$, Süßholz, Melonensamen und Graswurzeln ${ }^{17}$ mit Zucker und Pfirsichkernen.

(Am Rande des Blattes beigefügt: Andere empfehlen Kirschkerne und Gumi ? [möglicherweise Gemme = Harz des Kirschbaumes.])

Im Winter esse man in der Frühe an Stelle von Cassia als Electarium eine gewisse Menge Baumnüsse mit klarem Lärchenharz, Markpulver der Mespili ${ }^{18}$ und beigemengtem, nicht süßem Fenchelsamen.

${ }^{13}$ Serapion, Senex aus Alexandria um 200 v. Chr.; Gründer der empirischen Arzteschule, Nachfolger des Philinos. Galen, Celsus und Caelius Aurelianus führen ihn in ihren Schriften an.

${ }^{14}$ Die Cassia-Arten, Folia Sennae, werden heute noch als Laxans verwendet.

${ }^{15}$ Resina Laricis (= Lärchenharz) gilt wie alle Harze sekretionsfördernd. GrRolamo CARDANo berichtet in seiner Vita propria, daß er seine Dysurie mit Tannenharz behandelt habe H. HeFele p. 19).

${ }^{16}$ Cicer arietinum L.: Pois chiche, Kichererbse, Pisos Mauricos, Cicer punicum, C. italicum; als Kaffeesurrogat: "Café de France». Wilder Cicer schickte der Lyoner Arzt DaleChampius an C. Gessner (D. Fretz, K. Gessner als Gärtner, Zürich 1948, p. 214); wirkt diuretisch.

${ }^{17}$ Radices Graminis: bestehend vorwiegend aus Quecke, Graswurz = Agropyrum repens L.; als Aufguß erfrischend, harntreibend und leicht abführend.

${ }^{18}$ Mespili: Mespilus germanica L., Nespelbaum, Rosaceae. C. Gessner kannte den Mespilus tricoccus (D. Fretz K. Gessner als Gärtner. Zürich 1948, p. 246). 
Indem man alles Obgenannte, was die Hitze bewirkt, meidet, wird auch die Erwärmung der Nieren verhindert.

Täglich salbe man die Nierengegend mit Öl von Veilchen ${ }^{19}, K_{\text {ürbis }}^{20}$, Seerosen ${ }^{21}$ und mehrere Male mit der weißen Salbe des GALEN ${ }^{22}$.

Dies sind zur Verhütung nützliche und bewährte Vorschriften. Wenn der Stein wirklich da ist und durch Schmerzen beunruhigt, erfordert das eine Behandlung, von der ich im Augenblick nicht schreibe, etc.

(Darunter stehen neben einigen nicht zu entziffernden Worten: Bäder, Klystiere, Schröpfköpfe.)

\section{Soweit D. CAmutius.}

Eine biographische Notiz über Francesco Camuzio blieb unauffindbar, weder in der Bibliotheca universalis GESSNERS ${ }^{23}$, noch in der Epitome ${ }^{24}$, noch in den Briefen Gessners an Kentmann ${ }^{25}$, wiewohl dies möglich wäre, zumal Joh. Kentmann das kleine Büchlein Calculorum qui in corpore humano nascuntur (Tiguri 1565) veröffentlichte.

Die Bibliotheca universalis und der Epitome vermerken nur Andrea $\mathrm{CAmuzio}^{26}$. Dagegen trägt das Consilium Vadians ${ }^{27}$ schon einleitend den

${ }^{19}$ Oleum Violarum: aus Viola odorata L. früher auch als Sirupus violaceus eingenommen; Veilchen wirken auswurfbefördernd, ähnlich der Wirkung der Radix Ipecacuanhae (Emetin), leicht brechenerregend.

${ }^{20}$ Oleum aus Semina Cucurbitae $=$ Colocinthis officinalis ScHRADER $=$ Cucumis colocinthis L., Cucurbitaceae. Kürbissamen als ein heftiges drastisches Purgiermittel früher gegen Bandwürmer sehr geschätzt. Gessner nennt Cucurbita silvestris (Coloquinten = colocynthis, der wilde Kürbis) (D. Fretz, K. Gessner als Gärtner, Zürich 1948, p. 218).

${ }^{21}$ Oleum Nemofarini = Nenuphar oder Nymphaea alba major (Lys d'eau), Seerose als Mucilago, wirkt sedativ und antiaphrosialisch.

${ }^{22}$ Ungentum album GALENI = Ceratum refrigerans GALENI.

${ }^{23}$ Conr. GeSNer, Bibliotheca universalis, s. catalogus omnium scriptorum locupletissimus, in tribus linguis, lat. gr. et hebr. vet. et recent. publicatorum et in bibliothecis latentium, Tiguri 1545, Fol.

${ }^{24}$ Conr. Gesner, Epitome bibliothecae, primum a Conr. Lycosthene conscripta, nunc denuo recognita et insigniter aucta per Josiam Simlerum, Tiguri 1555, Fol.

${ }^{25}$ Joн. K. Kentmann, geb. in Dresden 1518, studierte 1536 in Leipzig, 1547 in Pavia, Stadtarzt in Dresden 1549/50, Stadtphysikus zu Torgau 1554, starb 1574, war mit C. Gessner befreundet.

${ }^{26}$ Bibl. universalis, p. 39: Andreas Camutius medicus in Gallia cisalpina aedisit propositiones quasdam in philosophia et medicina, quibus se responsurum pollicetur: et opusculum quoddam in quo philosophiam et theologiam conciliare conatur. - Epitome, p. 10: Andreas Camutius medicus in Gallia cisalpina, edidit propositiones quasdam in philosophia et medicina et opusculum quoddam in quo philosophiam et theologiam conciliare conatur.

${ }^{27}$ B. MrLt, Ein Konsilium Vadians. Schw. Med. Wschr. 62. Jg. (1932).

12 
Hinweis: «nit mit so gar strenger ordnung als Doctor CAMUTIUS meldet.» Dies deutet ebenfalls auf den besagten Sammler, der das Consilium FraNcesco Camuzios kannte.

Daß Gessner dieses Abschriftblatt Camuzios in den Händen hatte, bezeugen seine eigenen handschriftlichen Rand- und Fußnotizen.

Da bei entsprechender Lebensweise und Diät der meist günstige Verlauf des Steinleidens längst erwiesen war, versuchten die Ärzte die Steinbildung mit Brech- und Purgiermitteln zu verhüten oder die Austreibung des Steines mit Diuretica zu fördern.

Camuzio erwähnt ausführlich die Theorie Serapions über die Steinbildung, die der modernen patho-physiologischen Auffassung nicht allzu ferne steht, wenn sie von intermediären Abbaustörungen, besonders des Cystins, spricht.

Wenn auch die Druckerschwärze uns kein Werk F. CAMuzios übermittelte, die sein Sohn Andreas in so reichlichem Maße nützte, so mutet diese Mitteilung zur Verhütung des Steinleidens heute noch in seiner Denkweise recht vernünftig an und bekundet praktische Erfahrung in prophylaktischer wie therapeutischer Hinsicht aus erlebter ärztlicher Tätigkeit. Als Zeuge des leidvollen Menschen im Steinleiden vergaß dieser Luganeser Arzt keineswegs die psychische Seite des Krankseins, wenn er vor Zorn, Wut und Traurigkeit warnte.

Der Zufall will es, daß sowohl das Consilium Vadians wie dasjenige des Francesco Camuzio die einzigen Konsilien sind, die in Abschriften auf uns gekommen, was auch dieses mitzuteilen Berechtigung gibt. 